\title{
Isoflurane Impairs Motor Function Recovery by Increasing Neuroapoptosis and Degeneration during Spinal Ischemia-Reperfusion Injury in Rats
}

\author{
Chen-Fuh Lam, MD, PhD \\ Department of Anesthesiology, Buddhist Tzu Chi General Hospital, Hualien, Taiwan
}

INTRODUCTION. Spinal cord ischemia is one of the major concerns of postoperative paraplegia following major vascular or aortic surgery. The incidence of postoperative paraplegia during aortic surgeries is reported to be $0.5 \%-1.5 \%$ for coarctation repair, $10 \%$ for thoracic aneurysm repair, and up to $20 \%$ for thoracoabdominal aorta repair. Isoflurane is one of the most commonly used inhalation agents in major cardiovascular surgery and it has been shown to produce

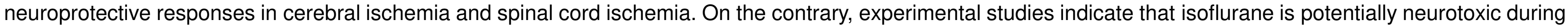
ischemia-reperfusion injury (IRI) and in cognitive disorders by generation of neurotoxic peptides. Therefore, the effects of perioperative administration of volatile

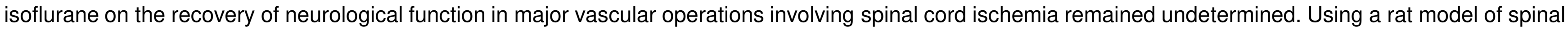
cord IRI, we analyzed the functional recovery and molecular changes in the spinal cord.

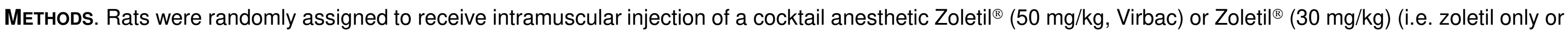

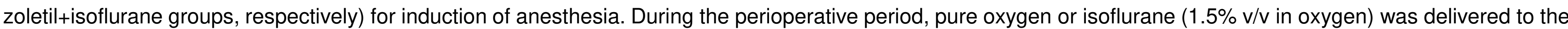

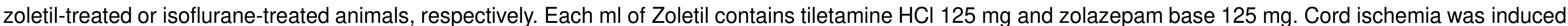
by cross-clamping of thoracic aorta at the level of T5, and cord perfusion was resumed after 25 min. Cessation of blood flow in the lumbar cord was confirmed by absence of Evans blue dyne following systemic perfusion (Figure 1). The motor function [Basso-Beattie-Bresnahan (BBB) scale (range 0-21)] was assessed independently up to $48 \mathrm{~h}$ after reperfusion. Spinal cords were harvested and analyzed for molecular and histological changes.

RESULTS. Six hours after release of aortic clip, the BBB locomotor rating scales were reduced in the hindlimbs of animals with spinal IRI (Figure 2), suggesting the presence of motor dysfunction due to ischemic cord syndrome. The locomotor function was gradually recovered in the rats received only Zoletil ${ }^{\circledR}$, and was almost returned to high BBB rating scale $(>16)$ at $48 \mathrm{~h}$ (Figure 2$)$. However, the locomotor function was significantly deteriorated $(<5)$ in the isoflurane $+Z$ Zoleti ${ }^{\circledR}$ group, and most of them eventually became paralysis at $48 \mathrm{~h}$ after operation (Figure 2). In animals treated with isoflurane, the expression of iNOS was suppressed and HO-1 was up-regulated in the spinal cord after SCI (Figure 3). Compared with controls, the concentration of IL-6 was reduced in the spinal cord of isoflurane-treated rats

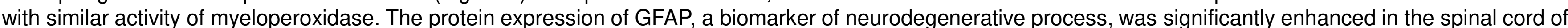

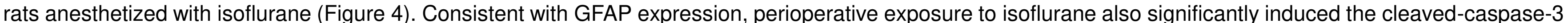

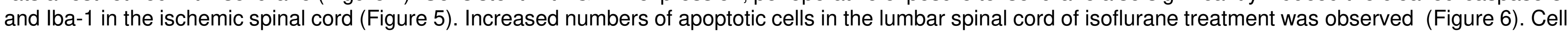
markers of neuronal degeneration (GFAP, caspase-3 and lba-1) were highly expressed in the spinal cord of rats anesthetized with isoflurane (Figure 7A). In the spinal cord subjected to IRI, more damaged motor neurons were identified in the ventral horn of grey matter of rats received isoflurane anesthesia (Figure 7B). Histological sections did not show increased infiltration of inflammatory cells in the spinal cord of isoflurane-treated animals (Figure 7B)

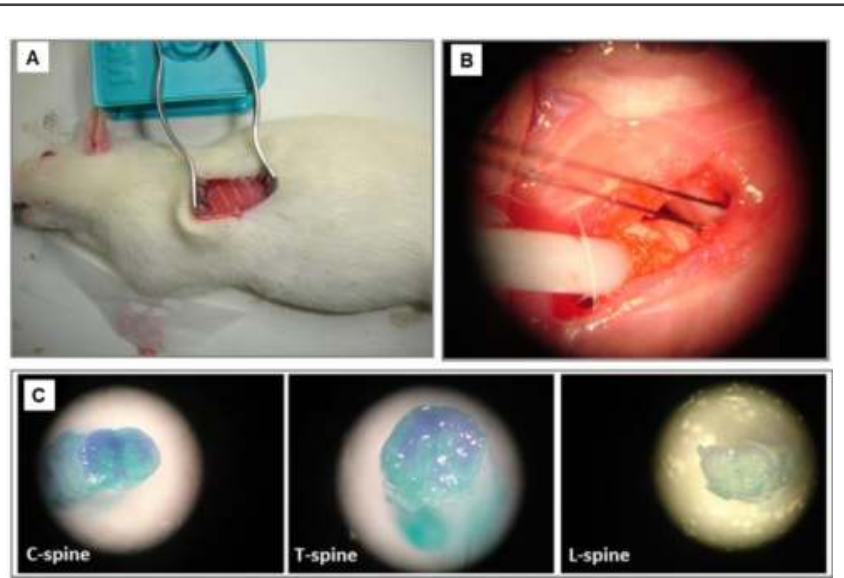

Figure 1. Rat model of spinal cord ischemia. Thoracic cavity was exposed at the left T5-6 intercostal space (A \& B) and vascular clip was applied to the thoracic aorta to induce hypoperfusion in the lumbar spine. Complete abolish of blood flow was confirmed by absence of Evans blue dye following systemic perfusion (C)

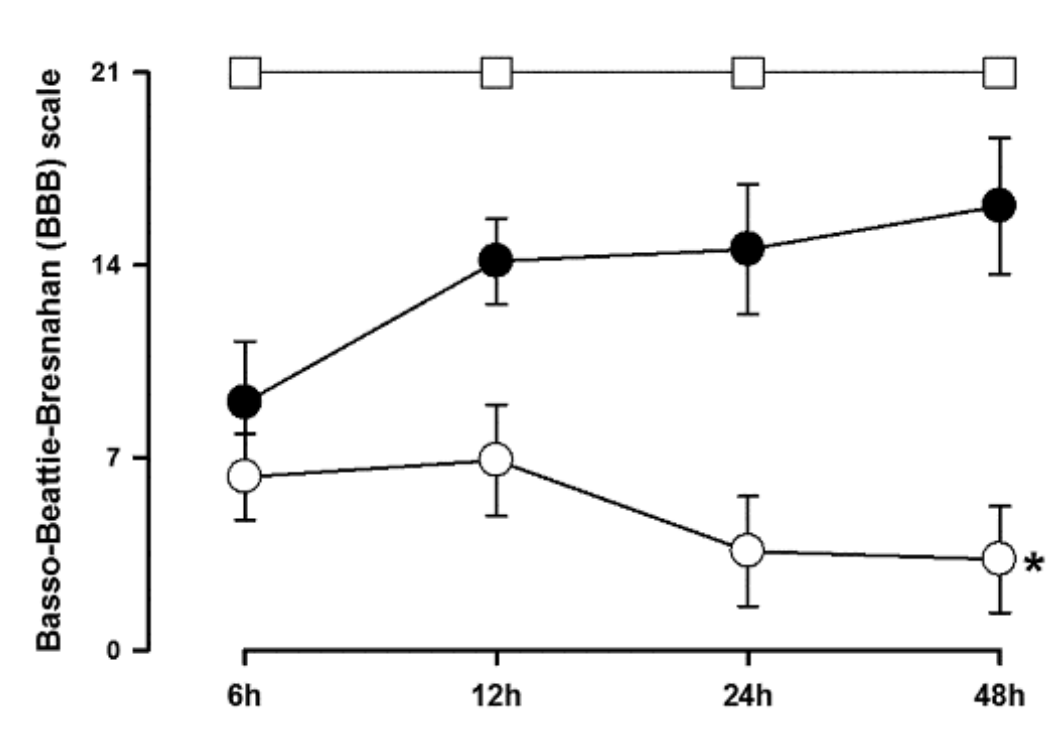

Figure 2. The BBB scale (range 0-21) was used to assess the hind limb locomotor function after reperfusion. Compared with Zoletil $(\mathbf{O})$, the BBB scales were significantly lower in the isoflurane-treated animals (○). Open boxes $(\square)$ indicate naïve treatment. ${ }^{*} P<0.001$ Zoletil vs isoflurane. $n=7-10$ in each group.
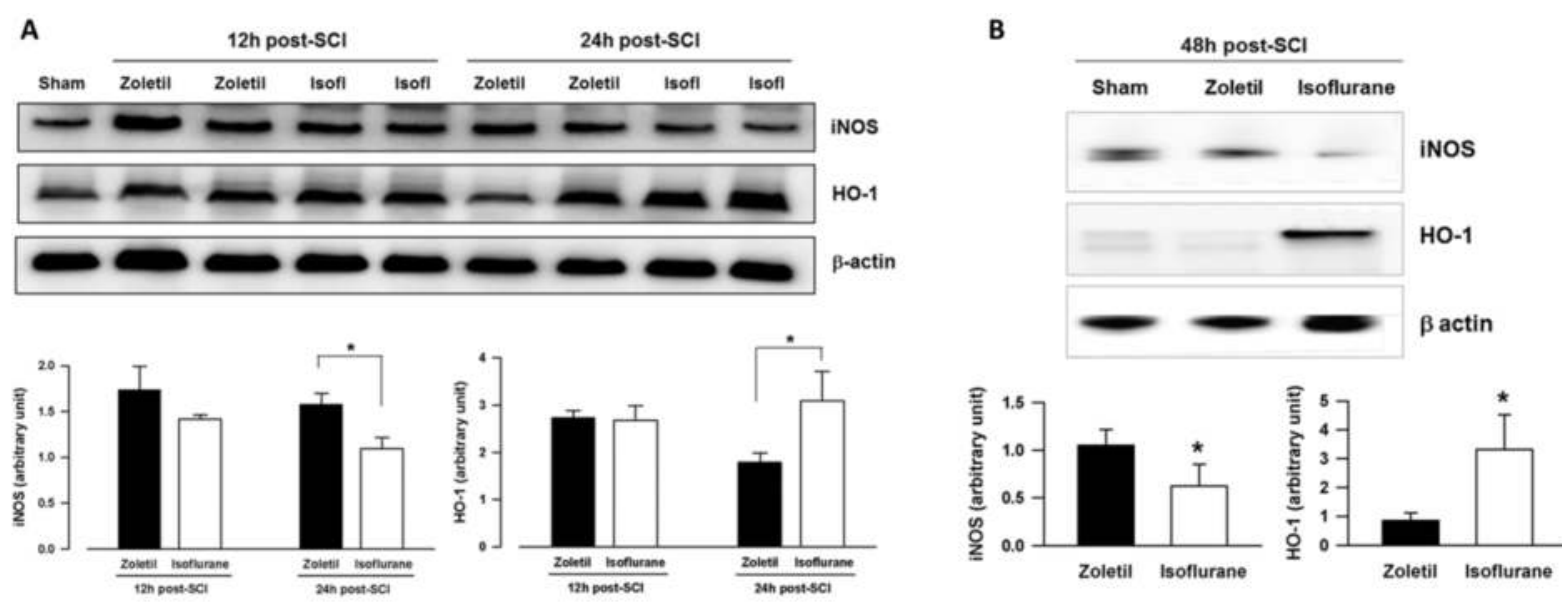

Figure 3. Protein expressions of inducible nitric oxide synthase (iNOS) and heme oxygenase $(\mathrm{HO})-1$ in the tissue homogenates of spinal cord at $12-24 \mathrm{~h}$ (A) and $48 \mathrm{~h}(\mathrm{~B})$ after spinal cord ischemia $(\mathrm{SCl}) . .{ }^{*} \mathrm{P}<0.05$ Zoletil vs isoflurane. $n=5-6$ in each group.

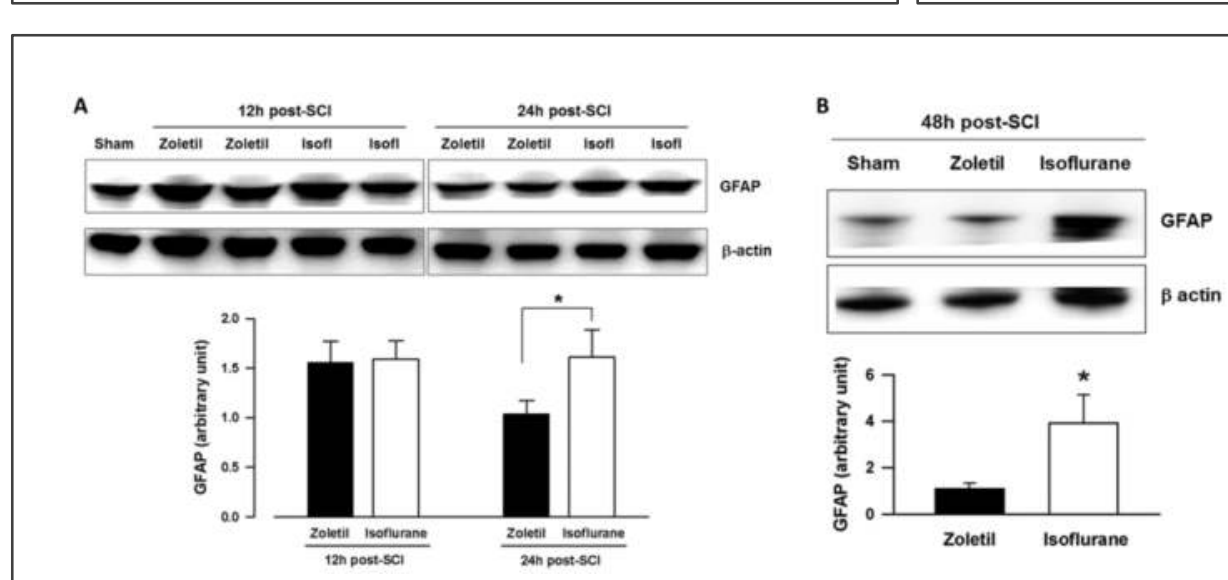

Figure 4. Expressions of glial fibrillary acidic protein (GFAP) in the spinal cord at $12 \mathrm{~h}-24 \mathrm{~h}$ (A) and 48h (B) after spinal cord ischemia $(\mathrm{SCl})$.. Data are presented as mean \pm SD. ${ }^{*} \mathrm{P}<0.05$ Zoletil vs isoflurane. $\mathrm{n}=5-6$ different animals.

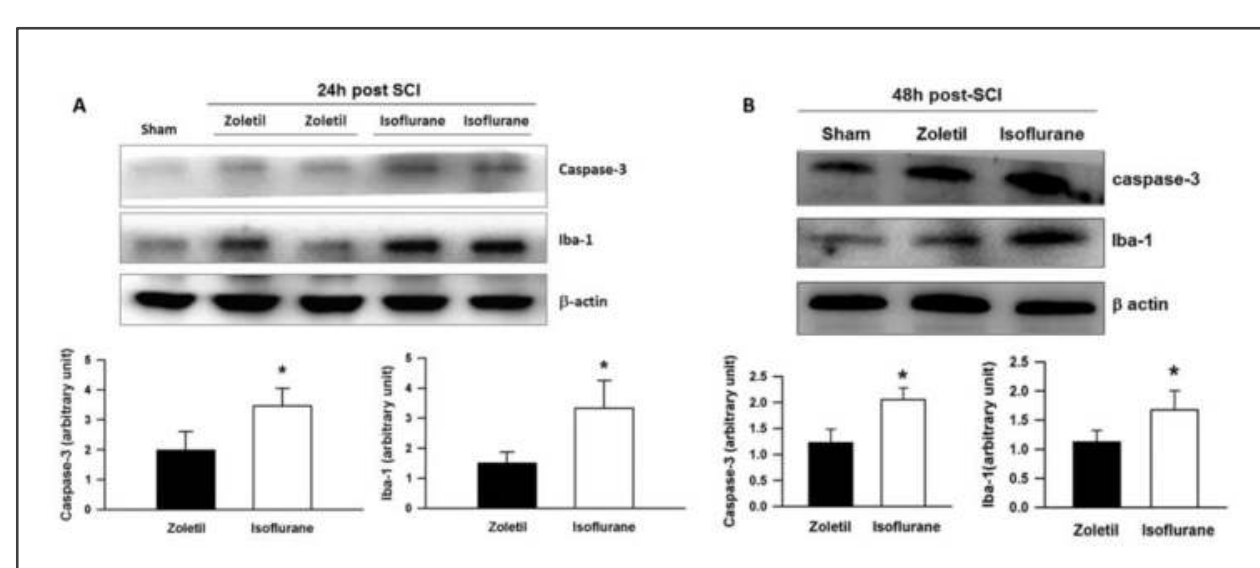

Figure 5. Expressions of cleaved caspase- 3 and lba- 1 in the spinal cord at $24 \mathrm{~h}(\mathrm{~A})$ and $48 \mathrm{~h}(\mathrm{~B})$ after spinal cord ischemia $(\mathrm{SCl})$. Data are presented as mean $\pm \mathrm{SD}$. ${ }^{*} \mathrm{P}<0.05$ Zoletil vs isoflurane. $n=5-6$ in each group.

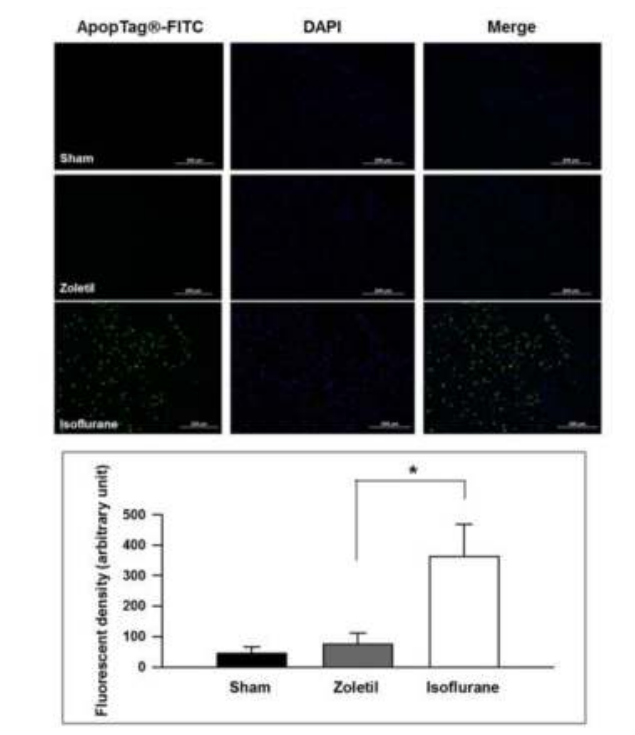

Figure 6. Significantly higher

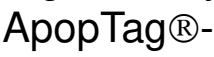
fluorescent densities were detected in the spinal cord harvested from isoflurane-treated rats. ${ }^{*} \mathrm{P}<0.001$ Zoletil vs isoflurane.
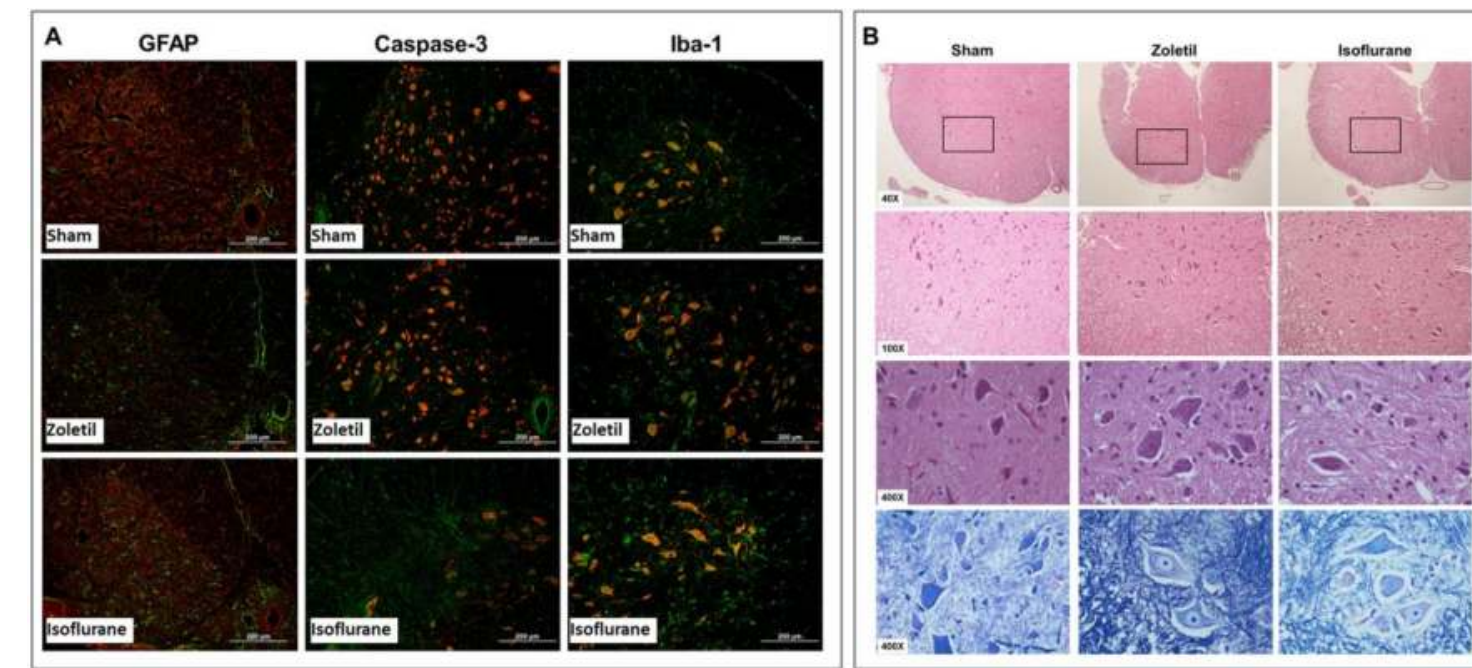

Figure . (A) Representative immunofluorescence sections of spinal cord stained for GFAP (green) and astrocytes S100 $\beta$ (red), caspase-3 (green)/ nuclei (neuN in red) and lba-1 (green)/neuN) (red). The neurodegnerative cell markers were highly expressed in the injured cord of rats anesthetized with isoflurane. (B) Histological sections by HE stain (upper 3 panels) and Nissl stain (last panel). Loosen Nissl substances, pyknotic homogenous nuclei and formation of vacuolation were observed in the ventral horn of grey matter of rats received soflurane anesthesia. $\mathrm{n}=3-4$ different animals in each group.

\section{CONCLUSION}

Our study demonstrated that pretreatment with isoflurane suppressed the regional inflammatory burden in the ischemic cord that was resemble with anesthetic preconditioning response, but the locomotor function was significantly impaired in these animals. The adverse effect of isoflurane on the regeneration of spinal cord subjected to IRI was derived from the neurodegenerative process and neuronal apoptosis. This study underscores that administration of isoflurane as an anesthetic agent in patients at risk of cord ischemia could result in detrimental effect on the functional recovery of spinal cord.

Acknowledgement. This study was supported by a research grant from the Buddhist Tzu Chi General Hospital (grant number TCRD104-27).

Contact. Chen-Fuh Lam, MD, PhD lamcf@mail.tcu.edu.tw 